\title{
The CryoLib - Modelling Superconductors with Modelica
}

\author{
Alexander Pollok Dirk Zimmer \\ Institute of System Dynamics and Control, DLR German Aerospace Center, Germany \\ \{alexander.pollok, dirk.zimmer\}@dlr.de
}

\begin{abstract}
A Modelica library for the thermal and electrical modelling of cryogenic and superconducting systems is presented. The library design is compatible with the electrical and fluid components from the Modelica Standard library. At the same time, several typical effects of cryogenic systems are modelled that have no equivalent in the Standard library. Easy usability and extendability of the components is emphasized. In this paper, the different parts of the library are explained in more detail. To illustrate the capabilities of the library, three showcases are presented: a simple solenoid magnet system, a current lead and a current limiter. The results show that many effects occuring in cryogenic and superconducting systems can successfully be simulated using this library.
\end{abstract}

Keywords: cryogenic, superconductivity, magnet, cryocooler, simulation, thermal, electric, HTS

\section{Introduction}

While the concept of superconductivity is known for over a century, it took a while for commercial applications to take root. In 2018, superconducting magnets can be found in the radiology departments of most first world hospitals. However, after all this time, the cost per kiloampere-meter for superconducting cables continues to go down, especially in the case of high temperature superconductors (HTS) (Selvamanickam et al., 2011; Rupich et al., 2013). This makes more and more applications viable. For example, cryogenic systems are envisioned for future electric aircraft projects (Gemin et al., 2015; Berg et al., 2015).

Thermal design makes up a big part of the overall effort that goes into the development of superconducting systems. For some applications like nuclear magnetic resonance tomography (NMRT) systems this can be done in Excel. In other applications, the transient behavior of the overall system is more important. This is especially true if the superconducting system is coupled to other systems and the overall system behavior cannot be neglected. In that case, Modelica is a naturally suited technology to describe the overall system behavior.

Some work has already been directed towards this topic: Łanczont (2016) presented a very detailed but also very specific electrothermal model of second generation HTS cables. A library for the simulation of supercritical helium loops is presented by Zanino et al. (2012) and verified in (Zanino et al., 2014). No general-use library for the typical aspects of the design of superconducting systems is available to best knowledge of the author.

This work closes that gap by presenting CryoLib, an easy-to-use Modelica library that provides components and interfaces to simulate the transient behavior of superconducting systems. The paper is structured as follows: In Section 2, the library structure is presented and some components are explained in more detail. Section 3 shows some examples that have been developed using the CryoLib library. These are used to present both physical effects and possible use cases of the library. The paper is discussed in Section 4 and concluded in Section 5.

\section{Library}

\subsection{Library Concept}

Based on the nature of the Modelica language, some usage scenarios for this library are more probable than others. If highly detailed three-dimensional effects have to be modelled, finite-element or similar methods should be used. For design or architecture studies, or for controller development, Modelica makes fast model development cycles possible, at the cost of accuracy. For this reason, the CryoLib is designed to be relatively light-weight and easy-to-use. Of course, an end-user can always choose to extend the library to their liking.

The library is divided into several sub-packages, see Figure 1 for the overall structure. The UserGuide subpackage contains general meta information about the library, the Examples package contains simulation models built from the library to demonstrate use cases. The other packages are presented in more detail in the following.

All component packages also contain a test-package. This is not only for validation of the components, but also serves as a tutorial to end users. 


CryoLib by DLR
Examples
Materials
PartialMaterial
Steel304
CopperRRR 100
NbTiCopper
Y... Tests

Conductors
SimpleCable
FluidChannelCable
Magnet
Motor
Tests

\section{ThermalBoundaries}

Radiation
Conduction
DryShield
Tests

\section{CryoCoolers}

\section{PulseTubeTwoStage}

- GiffordMcMahonOneStage

StirlingOneStage

Figure 1. Structure of the CryoLib

\subsection{Materials}

The Materials package contains several models that estimate material properties based on temperature, current density and magnetic field density. This is especially important since material properties can change significantly at low temperatures. For instance, the electric resistivity of very pure copper can be 100 to 1000 times smaller than the resistivity at room temperature.

The library includes technical high temperature superconductors, technical low temperature superconductors and non-superconductors like austenitic steel or copper. These models are also used as a basis for the components of the other sub-packages. For the superconducting materials, users can also change the ratio of superconductor to support medium using parameters.

Each of the models takes temperature, field and current as inputs, computing volumetric heat capacity, thermal conductivity and the electrical resistivity. The resistivity is set to zero if the material is inside the limits of superconductivity, based on field, temperature and current density. The dependency between these variables is not trivial and illustrated in Figure 2.

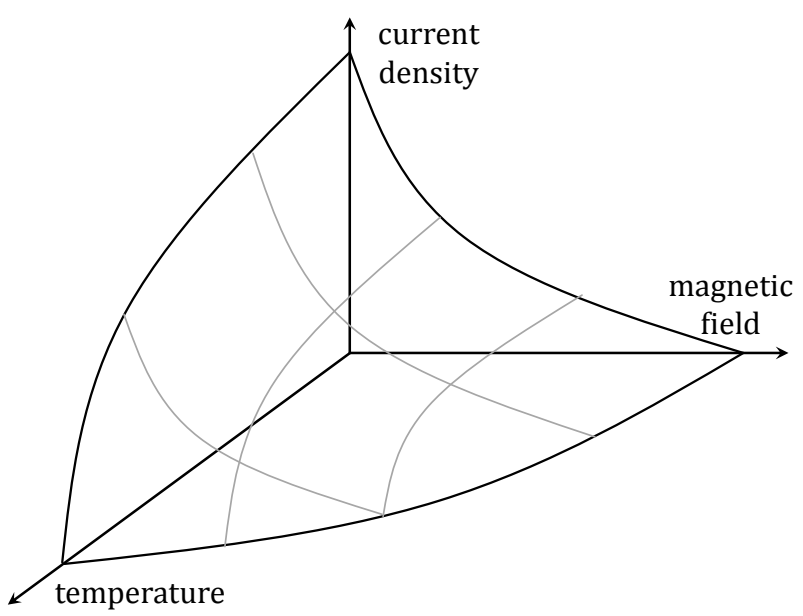

Figure 2. Illustration of the limits of superconductivity

The cryogenic data for the materials is taken from publicized manufacturer specifications (SuperPower Inc, 2014; AK Steel Corporation, 2007) and scientific papers (Bottura, 2000; Manfreda, 2011; Lu et al., 2008; Duthil, 2015). The data points are linearly interpolated afterwards.

While critical temperature and critical field are usually determined by the type of superconductor, critical current density is dependent on the manufacturing process. Therefore, the user can scale the critical surface in the direction of the current density using a parameter.

\subsection{Conductors}

This package contains several components that simulate superconductors. As such, it is the main package of the library. 
The most basic component is called ConductingElement. It models a cylindrical shape with axial electric current. The element material can be chosen from the Materials package. Two Modelica.Electrical.Analog connectors allow using the component similar to a conventional resistor element. Of course, the resistivity of the element can drop to zero if all requirements for superconductivity are met. As such, the overall electrical circuit has to allow for this possibility. Based on the voltage drop over the element, current, heat dissipation, temperature and maximum field is simulated. A connector from Modelica.Thermal.HeatTransfer allows for evacuation of dissipated heat.

The SimpleCable component models a discretized cylindrical shape. It behaves similar to multiple ConductingElement models stacked together.

The FluidChannelCable component behaves similar to the SimpleCable component, but additionally features Modelica.Fluid connectors. These can be used to model cable-in-conduit conductors (CICCs) or current leads.

The Magnet and Motor components are specialised versions of the ConductingElement component with additionally modelled inductance.

\subsection{ThermalBoundaries}

This package offers helper components to model typical thermal transfer effects in cryogenic systems.

In the Radiation component, thermal radiation is modelled based on the temperature on both sides, the area and the number of layers of used Multi-layer insulation. The Conduction and DryShield components can be used to simulate the transient thermal effects of radiation shields and the suspension.

\subsection{CryoCoolers}

Cryocoolers as described for instance by Satoh et al. (1996) are used to cool down small cryogenic systems. In the CryoCoolers subpackage, simple models representing several types of cryocoolers are included. These models offer a certain amount of cooling heat flow base on the temperature. Also, some amount of thermal inertia is included. The performance data is taken from $\mathrm{Xu}$ et al. (2008), Radebaugh et al. (2007) and Sunpower (2012).

\section{Examples}

In this section, several simulation models are shown that have been built using the Cryolib. Additionally, components from the Modelica Standard Library (ModelicaAssociation, 2008), HelmholtzMedia (Thorade and Saadat, 2012) and the NoiseLib (Klöckner et al., 2014) are used.

\subsection{Magnet System}

The first example system represents a standard superconducting magnet design with low temperature superconductors, as often used for nuclear magnetic resonance applications. It is illustrated in Figure 3.

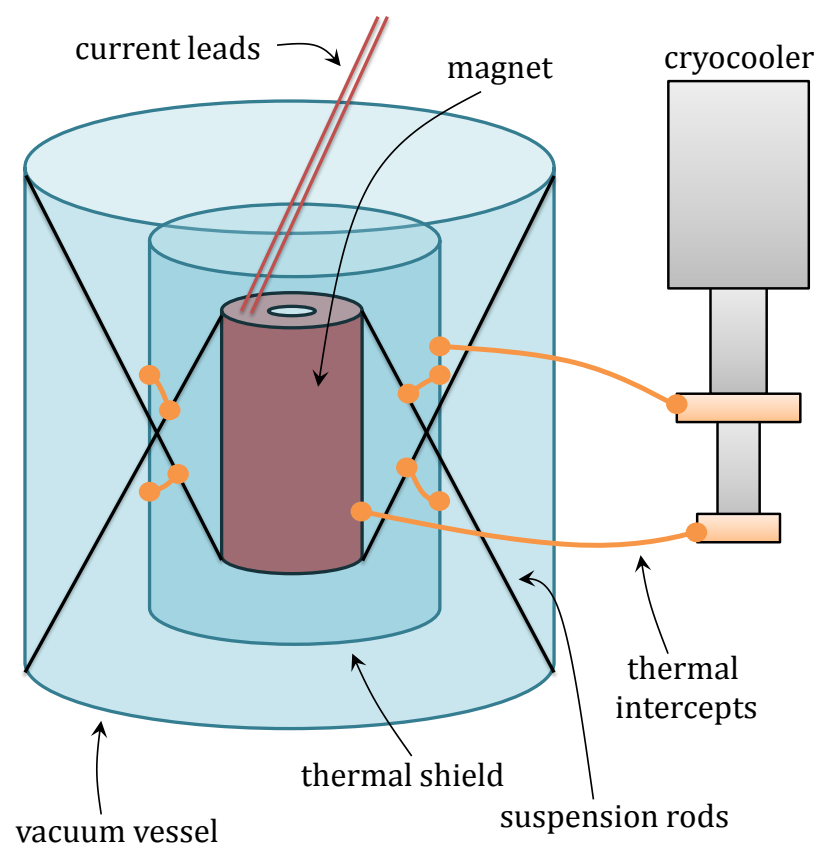

Figure 3. Illustration of a simple solenid magnet system

Refrigeration power is more costly at lower temperatures. For that reason, two-stage cryocoolers are used. These offer a small amount of refrigeration power at the operating temperature of the magnet (around 4 Kelvin) and a larger amount of refrigeration power at an intermediate temperature (around 80 Kelvin). A thermal shield is used to protect the cold side of the magnet from heat radiation and convection. The corresponding Modelica model is shown in Figure 4.

Conduction and Radiation elements are used to model the heat loads on both the shield and the actual magnet. Conduction elements are also used to model the heat resistance of the thermal intercepts between cryocooler, shield and magnet.

On the electrical side, a controller measures the magnet temperature. As soon as the temperature falls below 4.5 Kelvin, a constant voltage is applied until a target current is reached. The target current is set above the critical current, to force a quench. The thermal behavior of current leads is not modelled. The simulation results for this system are shown in Figure 5.

It is apparent that the shield side is colder than the magnet for the longest part of the cooldown. This can be explained by the smaller cooling power of the second stage compared to the first stage.

The magnet cooldown accelerates from 70 to $10 \mathrm{~K}$. This is due to the very small heat capacity of most materials at low temperatures. 


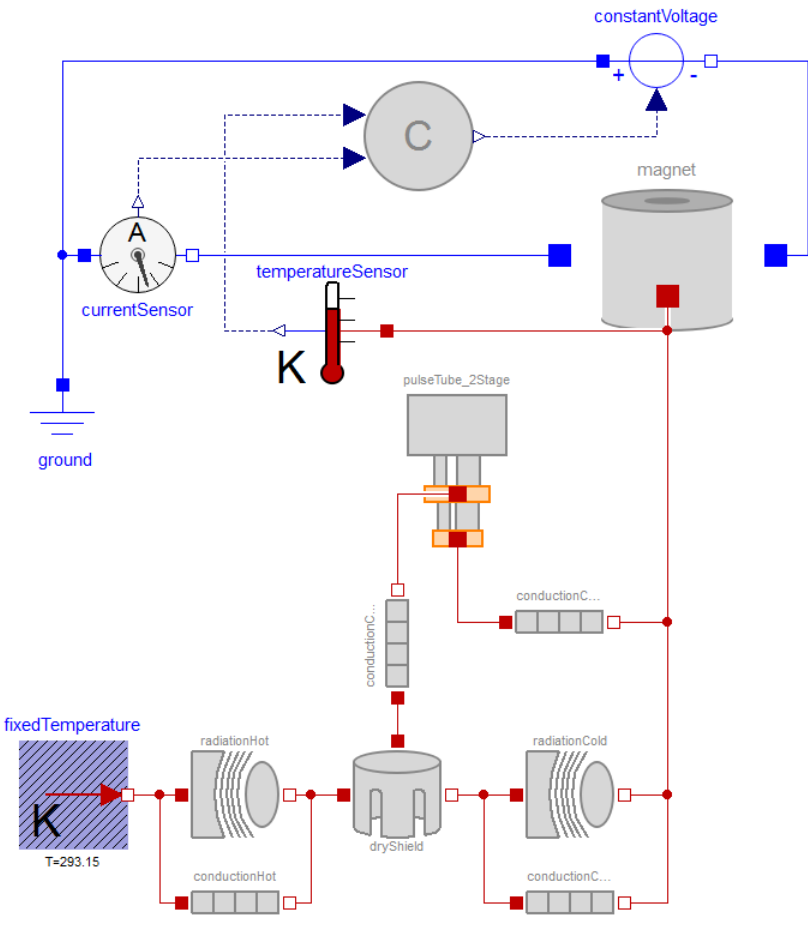

Figure 4. Modelica model of the "magnet system" example

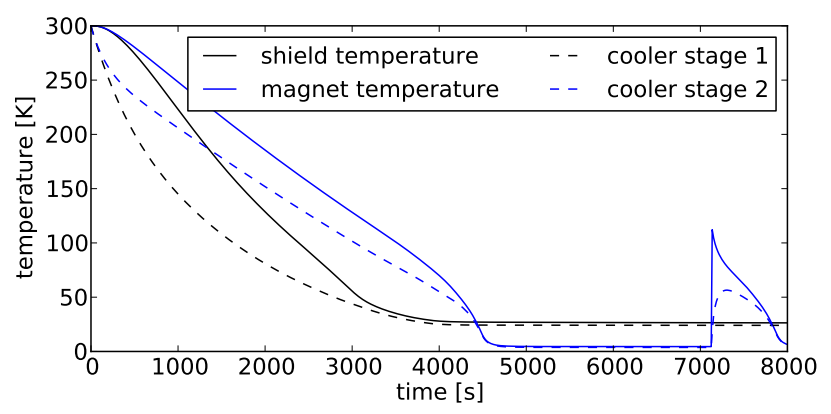

Figure 5. Simulation results for the "magnet system" example

At roughly 7000 seconds, a quench occurs. The magnet loses its superconducting property and the energy stored in the magnets inductivity is dissipated. This results in a sudden temperature spike.

\subsection{Current Lead}

The second example represents a current lead. Current leads are used to feed power from a conventional source to a cryogenic system. This generates a major engineering challenge, since electrical conductivity generally comes with unwanted thermal conductivity (Franz and Wiedemann, 1853) ${ }^{1}$. Also, the warm ends of current leads cannot be superconducting, generating additional electrical heat dissipation. These thermal loads can overwhelm most cooling systems, which have very low coefficients of performance at low temperatures.

\footnotetext{
${ }^{1}$ The original article is written in German. However, a search for "Wiedemann-Franz law" results in English translations.
}

A typical approach, which is also modelled here, is to counter-cool the current lead with helium. At the cold end, liquid helium is supplied into the current lead. It absorbs the majority of the dissipated heat and leaves the current lead at the warm end.

The modelled system is shown in Figure 6.

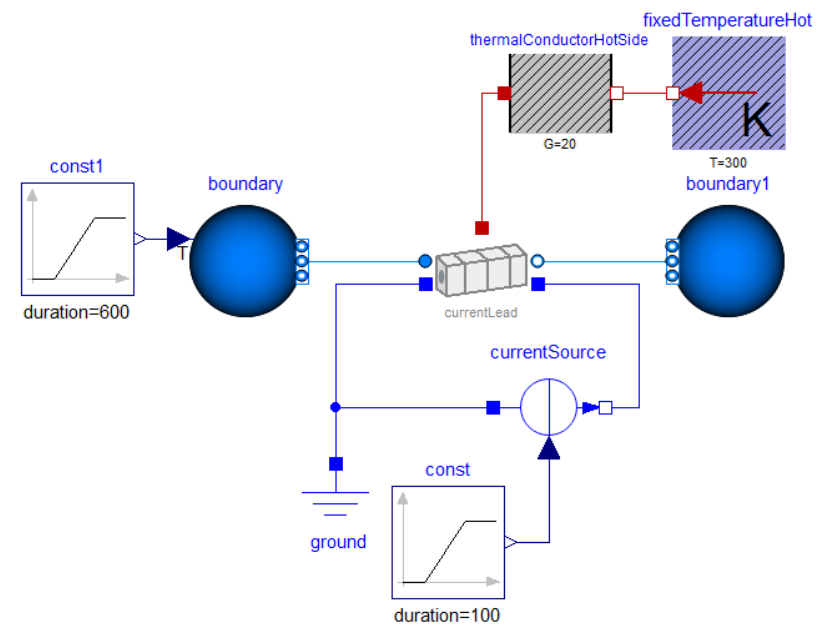

Figure 6. Modelica model of the "current lead" example

The current lead itself is modelled as copper with a residual resistivity ratio (RRR) of 100 and discretized into 6 elements. The system is initialized at room temperature and the helium source at the left is ramped down from 300 to 4 Kelvin in the first 600 seconds at slight overpressure. From 700 to 800 seconds, the current is ramped up from zero to 25.000 amperes. The right side of the current lead is connected to room temperature and atmospheric pressure.

The simulation results for this system are presented in Figure 7.

It can be seen that the temperature distribution in the current lead is not linear during the cooldown. This can be explained by the small thermal conductivity at low temperatures.

When the electrical current is ramped up from 700 to 800 seconds, a significant temperature increase can be seen throughout the current lead. The effect is much more pronounced near the warm side of the current lead. At low temperatures, the electrical conductivity of the used material is much better resulting in lower heat dissipation.

At 1000 seconds, the system is very near to the steady state. The axial heat flow in the current lead is 411 Watts at the warm end and 11.2 Watts at the cold end. That means that the cooling system only has to deliver 11.2Watts at a temperature of 4 Kelvin, in addition to the re-liquefaction of the boiled-off helium (5.5 grams per second).

Note that the used parametrization of the system was not optimized in any way. For benchmark designs, see for example Buyanov (1985). More advanced superconducting current lead designs are presented by Wesche and 

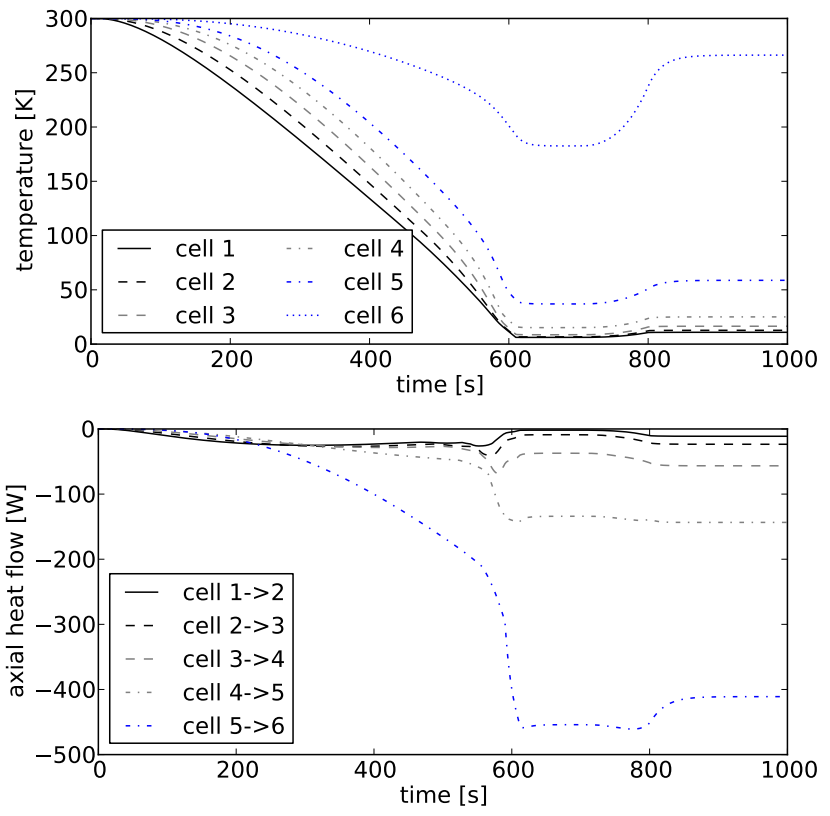

Figure 7. Simulation results for the "current lead" example

Fuchs (1994).

\subsection{Current Limiter}

Superconducting current limiters are used instead of fuses because they offer fast current shut-off and don't require maintenance after each triggering. High temperature superconductors are especially suited to this application. The modelled example shows a simple electric circuit with a variable resistor and inductivity. It is shown in Figure 8.

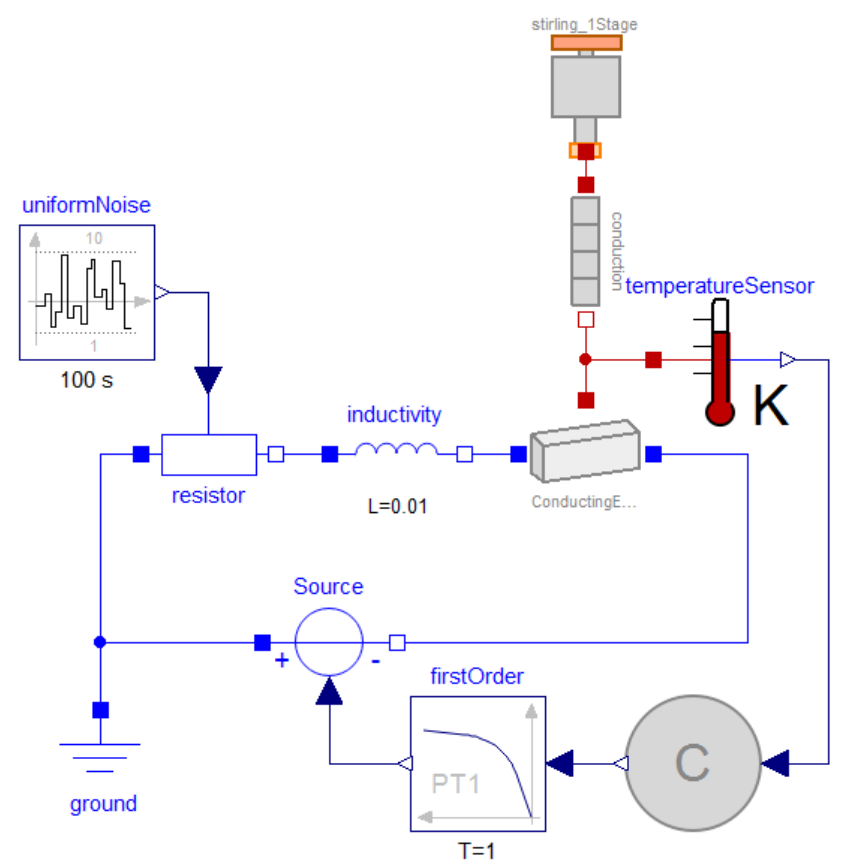

Figure 8. Modelica model of the "current limiter" example
A superconducting element is connected in parallel and cooled down using a cheap stirling-type cryocooler. A simple bang-bang controller switches on a constant voltage when the superconducting element is below 45 Kelvin and switches it off when it is above 100 Kelvin. The variable resistor is controlled by noise such that the maximum current of the superconducting element - which is also dependent on the temperature and the self-field - is sometimes exceeded. The simulation results can be seen in Figure 9.

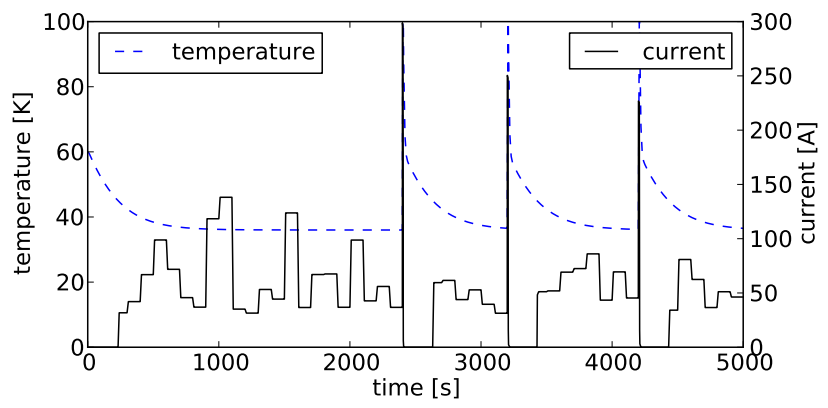

Figure 9. Simulation results for the "current limiter" example

The system reacts as designed. Every time the maximum current is exceeded, superconductivity is lost. The resistivity of the current limiter is now dominating the system and most of the energy stored in the inductivity is dissipated here. The cooler takes some time to cool the limiter down again and the cycle starts over.

\section{Discussion and Future Work}

The library as presented can be used as a basis for quick optimization studies, controller development or plausibility considerations. For more involved simulation tasks, extensions are necessary. For instance, a superconducting cable can not only be discretized in axial direction, but also in radial direction. This can be important since the self-induced magnetic field in an electrical conductor is linearly dependent on the distance to the central axis. The conductor can still be superconducting near the central axis when superconductivity is already lost near the border. Also at the moment, there is no anisotropy included for high temperature superconductors. These are often produced in the form of thin tapes. The field strength limits are much higher if the field vector is in the plane of the tape (Selvamanickam et al., 2011; Hazelton et al., 2009). Extensions of the library could include these effects, at the cost of added complexity.

\section{Conclusion}

We present a library for the modelling and simulation of transient cryogenic systems. The library can be used together with the Modelica Standard Library, while extending the range of modelled effects significantly. 


\section{Acknowledgements}

We thank Trey and Matt for inspiration.

\section{References}

AK Steel Corporation. 304 and 3041 stainless steel. Technical report, 2007. URL www.aksteel.com/pdf/ markets_products/stainless/austenitic/ 304_3041_data_sheet.pdf.

F Berg, J Palmer, L Bertola, Paul Miller, and Graham Dodds. Cryogenic system options for a superconducting aircraft propulsion system. In IOP Conference Series: Materials Science and Engineering, volume 101, page 012085. IOP Publishing, 2015.

Luca Bottura. A practical fit for the critical surface of nbti. IEEE transactions on applied superconductivity, 10(1):1054-1057, 2000 .

Yu L Buyanov. Current leads for use in cryogenic devices. principle of design and formulae for design calculations. Cryogenics, 25(2):94-110, 1985.

Patxi Duthil. Material properties at low temperature. arXiv preprint arXiv:1501.07100, 2015.

R Franz and G Wiedemann. Ueber die wärme-leitungsfähigkeit der metalle. Annalen der Physik, 165(8):497-531, 1853.

Paul Gemin, Tom Kupiszewski, Arthur Radun, Yan Pan, Rixin Lai, Di Zhang, Ruxi Wang, Xinhui Wu, Yan Jiang, Steve Galioto, et al. Architecture, voltage, and components for a turboelectric distributed propulsion electric grid (avc-tedp). 2015.

Drew W Hazelton, Venkat Selvamanickam, Jason M Duval, David C Larbalestier, William Denis Markiewicz, Hubertus W Weijers, and Ronald L Holtz. Recent developments in $2 \mathrm{~g}$ hts coil technology. IEEE Transactions on Applied Superconductivity, 19(3):2218-2222, 2009.

Andreas Klöckner, Franciscus LJ van der Linden, and Dirk Zimmer. Noise generation for continuous system simulation. In Proceedings of the $10^{\text {th }}$ International Modelica Conference, number 96, pages 837-846. Linköping University Electronic Press, 2014.

Michał Łanczont. Electro-thermal numerical model of superconducting tape. Przeglad Elektrotechniczny, 92(6):134-137, 2016.

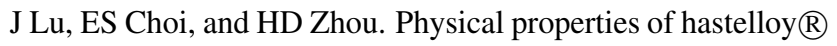
c-276 at cryogenic temperatures. Journal of applied physics, 103(6):064908, 2008.
Giulio Manfreda. Review of roxie's material properties database for quench simulation. TE Technology department internal note, 35, 2011.

Modelica-Association. The Modelica Standard Library. Online, URL: http://www.modelica.org/libraries/Modelica, 2008.

Ray Radebaugh, Agnes O'Gallagher, Michael A Lewis, and Peter E Bradley. Proposed rapid cooldown technique for pulse tube cryocoolers. Cryocoolers 14, edited by SD Miller and RG Ross, Jr, pages 231-240, 2007.

Martin W Rupich, Xiaoping Li, Srivatsan Sathyamurthy, Cornelis LH Thieme, Kenneth DeMoranville, John Gannon, and Steven Fleshler. Second generation wire development at amsc. IEEE transactions on applied superconductivity, 23 (3):6601205-6601205, 2013.

Toshimi Satoh, Atsushi Onishi, Rui Li, Hiroshi Asami, and Yoshiaki Kanazawa. Development of $1.5 \mathrm{w} 4 \mathrm{k}$ gm cryocooler with magnetic regenerator material. In Advances in cryogenic engineering, pages 1631-1637. Springer, 1996.

V Selvamanickam, Y Chen, I Kesgin, A Guevara, T Shi, Y Yao, Y Qiao, Y Zhang, G Majkic, G Carota, et al. Progress in performance improvement and new research areas for cost reduction of $2 \mathrm{~g}$ hts wires. IEEE Transactions on Applied Superconductivity, 21(3):3049-3054, 2011.

Sunpower. Cryotel gt. Technical report, 2012. URL http://sunpowerinc.com/cryocoolers / cryotel-family/gt/.

SuperPower Inc. Superpower $2 \mathrm{~g}$ hts wire specifications. Technical report, 2014. URL http: //www. superpower-inc.com/system/files/ SP_2G+Wire+Spec+Sheet_2014_web_v1.pdf.

Matthis Thorade and Ali Saadat. Helmholtzmedia - a fluid properties library. In Proceedings of the 9th International MODELICA Conference; September 3-5; 2012; Munich; Germany, number 076, pages 63-70. Linköping University Electronic Press, 2012.

$\mathrm{R}$ Wesche and Am M Fuchs. Design of superconducting current leads. Cryogenics, 34(2):145-154, 1994.

M Xu, H Takayama, and K Nakano. Development of high efficiency $4 \mathrm{k}$ two-stage pulse tube cryocooler. Georgia Institute of Technology, 2008.

Roberto Zanino, Roberto Bonifetto, Laura Savoldi Richard, and Francesco Casella. Dynamic modeling of a supercritical helium closed loop with the $4 \mathrm{c}$ code. In AIP Conference Proceedings, volume 1434, pages 1743-1750. AIP, 2012.

Roberto Zanino, Roberto Bonifetto, C Hoa, and L Savoldi Richard. Verification of the predictive capabilities of the $4 \mathrm{c}$ code cryogenic circuit model. In AIP conference Proceedings, volume 1573, pages 1586-1593. AIP, 2014. 